\title{
Autour de la guerre totale
}

David Bell, Annie Crépin, Hervé Drevillon, Olivier Forcade et Bernard Gainot

\section{(2) OpenEdition}

\section{Journals}

Édition électronique

URL : https://journals.openedition.org/ahrf/12236

DOI : $10.4000 /$ ahrf. 12236

ISSN : 1952-403X

Éditeur :

Armand Colin, Société des études robespierristes

Édition imprimée

Date de publication : 1 décembre 2011

Pagination : 153-170

ISSN : 0003-4436

\section{Référence électronique}

David Bell, Annie Crépin, Hervé Drevillon, Olivier Forcade et Bernard Gainot, « Autour de la guerre totale ", Annales historiques de la Révolution française [En ligne], 366 | octobre-décembre 2011, mis en ligne le 01 décembre 2014, consulté le 01 juillet 2021. URL : http://journals.openedition.org/ahrf/ 12236 ; DOI : https://doi.org/10.4000/ahrf.12236 


\title{
AUTOUR DE LA GUERRE TOTALE
}

\author{
David BELL \\ Annie CRÉPIN \\ Hervé DRÉVILLON \\ Olivier FORCADE \\ Bernard GAINOT
}

La notion de guerre totale est liée initialement au contexte des lendemains de la première guerre mondiale; la thématique, chez Léon Daudet (1918) ou chez le maréchal Ludendorff (1936) est plutôt celle d'un effort de guerre sans précédent, compte tenu de la mobilisation de l'appareil industriel.

Toutefois, la dimension idéologique est à la base de l'ouvrage de Ludendorff puisque ce dernier prône un «peuple total » - épuré racialement - seul apte à conduire une «guerre totale». Mais la reprise de la notion dans le champ historiograhique, par George Mossé d'abord, puis par Stéphane Audoin-Rouzeau, lui confère une dignité et une actualité problématiques. Cette fois, c'est plutôt la dimension anthropologique de la violence guerrière qui est en cause, ainsi que le transfert de l'expérience de la brutalisation du combat vers la société civile.

Ainsi, à travers l'élaboration de ces problématiques, la notion de guerre totale tend à se généraliser à d'autres époques et à d'autres guerres, notamment celles de la Révolution et de l'Empire. Deux ouvrages récents en portent témoignage; celui de Jean-Yves Guiomar (L'invention de la guerre totale), celui de David Bell (La première guerre totale). Cette extorsion de son contexte initial pour l'étendre à d'autres périodes estelle justifiée, dans la mesure où les phénomènes en jeu paraissent emblématiques de la Grande Guerre - la mobilisation de masse, le contrôle de la guerre sur l'ensemble de l'appareil de production industriel, la propagande de guerre, la représentation de l'ennemi et le caractère illimité des 
buts de guerre, le lien entre la Nation, la société civile et le commandement militaire, etc.?

L'aspect méthodologique de ce transfert d'une période à une autre doit être interrogé, toute analogie de façade pouvant se révéler trompeuse, voire à l'usage porteuse de contresens; ainsi la mobilisation patriotique, par la routine de la conscription, peut-elle être vecteur de diffusion des valeurs guerrières dans le corps social. Guibert avait anticipé à la fin du XVIII ${ }^{\mathrm{e}}$ siècle le fait que la généralisation du recrutement à l'ensemble de la Nation portait le risque de conflits illimités. Peut-on pour autant considérer que la nature du conflit qui commence en 1792 est fondamentalement différente de la nature de ceux qui l'ont précédé?

C'est aussi s'interroger sur la pertinence des découpages chronologiques, et les risques d'anachronisme qu'ils induisent. La sélection de la période révolutionnaire par rapport à l'amont conduit une fois de plus, mais dans un sens négatif, à valoriser la coupure révolutionnaire par rapport aux guerres $\mathrm{du} \mathrm{XVIII}^{\mathrm{e}}$ siècle. Certes, tout un courant historiographique, emboîtant le pas à Carl Schmidt et à son insistance sur la déstabilisation du jus publicum europeum, évoque avec nostalgie la fin, sinon de la « guerre en dentelles », du moins de la " guerre réglée ». N'est-ce pas là une parfaite illusion, les guerres patriotiques ne datant pas de l'époque révolutionnaire, d'une part, les combattants de la Révolution et de l'Empire se référant toujours aux principes du droit de la guerre et des gens, d'autre part? La relativisation de la césure historique conduit également à regarder vers l'aval, vers le saut véritable de l'appareil militaire dans l'âge industriel, et à prendre en considération la nature des conflits du XIX ${ }^{\mathrm{e}}$ siècle, tout particulièrement la guerre de Sécession américaine.

Toute opération de découpage ayant des présupposés idéologiques, que faut-il en penser en l'occurrence pour cette période révolutionnaire et impériale? N'est-ce pas imputer aux seuls révolutionnaires français la responsabilité d'une croisade idéologique sans fin, qui aurait fait basculer dans la terreur la civilisation policée des Lumières? Cette lecture unilatérale des origines de l'entrée en guerre de l'Europe en 1792 connaît un regain récent de publicité.

Nous avons demandé à David Bell, professeur à l'université de Princeton, l'un des protagonistes majeurs de ce débat, à Olivier Forcade, professeur à l'université Paris IV, spécialiste des conflits contemporains, et à Hervé Drévillon, professeur à l'université Paris I, spécialiste de la guerre à l'époque moderne, de partager ces réflexions et ces 
interrogations. Qu'ils soient tous trois remerciés pour leurs réponses argumentées.

Annie CRÉPIN et Bernard GAINOT

Comment définir la guerre totale, dans la mesure où cette notion n'est jamais employée par les acteurs de la fin du XVIII siècle? Quel est l'intérêt, mais aussi quels sont les dangers, à transposer les analyses très contextualisées de Léon Daudet et Ludendorff vers les guerres révolutionnaires et impériales?

De quelle guerre totale parle-t-on? Quel est le rapport entre guerre totale et guerre absolue?

\section{David A. BELL}

Il est à noter qu'à l'origine (en 1917-18), le terme de guerre totale n'est pas employé pour donner une description fidèle d'un état de guerre réelle. Ceux qui en parlent la définissent généralement comme la mobilisation de toutes les ressources humaines et matérielles d'une nation pour détruire totalement une nation ennemie, sans faire de distinction entre des militaires et des civils. Cependant, ils avouent que ce niveau de mobilisation n'est pas encore atteint. L'idée de guerre totale sert plutôt pour appeler à un effort encore plus grand, un engagement encore plus profond, une mobilisation encore plus étendue. Autrement dit, elle représente quelque chose d' « idéal », voire un mythe dans le sens sorélien du mot. Goebbels lui-même, dans son célèbre discours du Palais des Sports de Berlin de 1943 (« Wollt ihr den totalen Krieg? »), présente la guerre totale comme un objectif qui reste encore à atteindre.

À cause de cette origine, le terme pose un problème évident pour tout historien qui pense l'utiliser comme une catégorie d'analyse historique. Cette guerre totale « idéale » conçue dans les imaginations fébriles du premier vingtième siècle, a-t-elle jamais été réalisée dans l'histoire? Peut-être en Allemagne et en URSS à la fin de la deuxième guerre mondiale, mais ailleurs, presque toujours, les sociétés s'effondrent avant de pouvoir arriver au niveau de mobilisation nécessaire - comme par exemple en Allemagne en 1918 -. Ainsi, pour que l'idée de guerre totale soit utile pour les historiens, ils ne peuvent se l'approprier sans la modifier, sans la définir de façon moins absolue, et plus large. C'est-à-dire qu'il leur faut tracer une ligne entre les guerres qui ne sont pas encore « totales » et celles qui s'approchent suffisamment de «l'idéal » pour en mériter l'étiquette.

Mais comment tracer cette ligne? Depuis une quinzaine d'années, une équipe d'historiens allemands, anglais et américains ont fait l'effort le 
plus systématique pour y parvenir, dans une série de six colloques, suivie de six volumes collectifs au sujet de la guerre totale dans l'histoire contemporaine ${ }^{1}$. Cependant, ils ne sont pas arrivés à un consensus, tant s'en faut, et l'un des principaux organisateurs avoue avoir des doutes quant à la possibilité de jamais trouver une ligne de démarcation satisfaisante ${ }^{2}$.

Depuis une dizaine d'années, quelques historiens - dont Jean-Yves Guiomar et moi-même - proposent que « la guerre totale » soit plus spécifiquement comprise comme un concept politique autant que militaire ${ }^{3}$. Comme l'observe Guiomar, ceux qui la prônent en 1917-18 sont plus souvent des leaders civils que des soldats, et ils imaginent une mobilisation dirigée par le gouvernement, et non par l'armée 4 .

Dans mon propre travail, je suggère que si l'idéal de la guerre totale ne peut se réaliser, sauf dans quelques cas exceptionnels, la poursuite de cet idéal par des états belligérants peut mener néanmoins à une radicalisation irrésistible et incontrôlable de la guerre, une course à l'abîme qu'il est presque impossible d'arrêter jusqu'à ce que l'une des parties s'effondre. Cette radicalisation est mise en marche par la politique : par la détermination des autorités civiles de se battre jusqu'au bout et par la diabolisation de l'ennemi - sa transformation dans les représentations en menace existentielle et inhumaine qui doit être détruite -. En outre, les guerres totales sont vues par ceux qui y sont engagés comme une rupture historique, voire apocalyptique, et non pas comme un élément ordinaire

(1) Tous les livres suivants sont publiés par Cambridge University Press et The German Historical Institute (Washington, DC) : On the Road to Total War : The American Civil War and the German Wars of Unification, 1861-1871, Stig FörSTER et Jorg NAGLER (dir.),1997; Anticipating Total War: The German and American Experiences, 1871-1914, Manfred F. BoEMEKe, Roger Chickering et Stig Förster (dir.), 1999; Great War, Total War : Combat and Mobilization on the Western Front, 1914-1918, Roger CHICKERING et Stig FöRster (dir.), 2001 ; The Shadows of Total War : Europe, East Asia, and the United States, 1919-1939, Id., (dir.), 2003; A World at Total War : Global Conflict and the Politics of Destruction, 1937-1945, Roger CHICKERING, Stig Förster et Bernd Greiner (dir.), 2005; War in an Age of Revolution, 1775-1815, Roger Chickering et Stig FÖRSTER (dir.), 2010.

(2) Roger Chickering, « Total War: The Use and Abuse of a Concept, » Anticipating Total War, p. 13-28.

(3) Jean-Yves Guiomar, L'invention de la guerre totale : XVIII -XX $X^{e}$ siècle, Paris, Le Félin Kiron, 2004; David A. Bell, The First Total War : Napoleon's Europe and the Birth of Warfare As We Know It, Boston, Houghton Mifflin, 2007; Id., La première guerre totale : l'Europe de Napoléon et la naissance de la guerre moderne, traduction française par Christophe Jaquet, Seyssel, Champ Vallon, 2010.

(4) Jean-Yves GuIOMAR, L'invention...op. cit., p. 302. Voir aussi l'article de Michael GeYer, «People's War: The German Debate About a Levée en masse in October 1918 », The People in Arms : Military Myth and National Mobilization since the French Revolution, Daniel Moran et Arthur Waldron (dir.), Cambridge, Cambridge University Press, 2003, p. 124-158. 
de l'ordre social. Donc domine la rhétorique de « la lutte finale» ou de « la guerre qui mettra fin à toutes les guerres » : par exemple, pour citer Dumouriez en 1792, « cette guerre-ci sera la dernière $»^{5}$.

Il existera toujours, bien entendu, des historiens qui s'opposeront à l'emploi d'un terme qui n'est pas connu à l'époque en question. Ils insisteront sur le fait que, pour les guerres de 1792 à 1815, il faut parler, tout au plus, de « guerre absolue », pour emprunter l'expression de Clausewitz (que le théoricien allemand définit surtout dans le deuxième chapitre du livre VIII de De la guerre $)^{6}$. Quant au premier point, je ne pense pas que les historiens puissent se limiter au vocabulaire employé par les sujets de leurs recherches - comment alors écrire l'histoire des Étrusques ou des Aztèques? Quant à Clausewitz, selon sa définition implicite, la «guerre absolue » consiste dans l'emploi de toutes les forces possibles au cours des opérations militaires pour arriver à un objectif défini par les autorités politiques (il donne, dans le troisième chapitre du livre VIII, l'exemple de Bonaparte et la Russie). Le point de repère essentiel pour cet officier prussien est la défaite écrasante de son pays par la France en 1806 - c'est cette campagne qui constitue pour lui l'exemple le plus pur de la guerre absolue. Par contre, la guerre totale, dans la définition politique que j'en donne, ne consiste pas seulement dans la réalisation, par les opérations militaires, d'un objectif politique, mais dans la définition même de cet objectif.

\section{Hervé DréviLlon}

L'historicité du concept de guerre totale est évidemment un problème crucial qui, d'ailleurs, ne se limite pas à la question de la contextualisation des analyses de Ludendorff et Daudet. La guerre totale a revêtu d'autres visages au $\mathrm{Xx}^{\mathrm{e}}$ siècle : massacres de masse, bombardements stratégiques conventionnels puis nucléaires. Dès lors, la possibilité même d'utiliser un concept unique pour évoquer les guerres de la Révolution et l'apocalypse nucléaire, perd tout son sens.

\section{Olivier ForCade}

Dans Clausewitz, la guerre, acte de violence d'un État voulant réduire un autre État à sa merci, est interprétée comme conduisant à

(5) Archives parlementaires, vol. LII, p. 472 (12 octobre 1792).

(6) Voir, par exemple, la recension très critique de mon livre par Annie Jourdan dans «H-France Forum », vol. II, numéro 3 (2007), et ma réponse : http://www.h-france.net/forum/ forumvol2/JourdanOnBell1.html et http://www.h-france.net/forum/forumvol2/Bell1Response.html 
l'anéantissement des seules forces militaires sur le champ de bataille. Ainsi commence la lecture de l'œuvre Vom Kriege de Clauzewitz par le général Ludendorff dans l'ouvrage que publie l'ancien chef d'état-major général allemand en 1937, La guerre totale (Flammarion, 1937). Plus sûrement que Daudet, Ludendorff date la naissance de la guerre totale de la Grande Guerre de 1914-1918 qui réalise, selon lui, la fusion entre l'armée et le peuple pour donner naissance à la guerre de masse dont il perçoit des prodromes côté français lors de la guerre franco-prussienne de 1870-1871. La combinaison des systèmes de recrutement des hommes, caractéristique de l'avènement de la démocratie et de l'organisation de la conscription universelle, avec l'évolution de systèmes d'arme innovants, s'appuyant sur une mobilisation industrielle, puis progressivement économique, et une bureaucratie de guerre, fait entrer dans une nouvelle échelle de la guerre. Cette échelle, à la fois géographique, temporelle, économique, mentale de la guerre mondiale explique que, sur l'ensemble du territoire d'un pays, la population combattante, y compris celle civile qui ne porte pourtant pas les armes des soldats, subit «l'action directe de la guerre » (La guerre totale, op. cit., p. 8). Cette dernière subit également ses manifestations indirectes par les effets du blocus (ici Ludendorff introduit la lecture allemande du blocus économique allié contre les puissances centrales, organisé au printemps 1915 et levé en juillet 1919) et ses effets directs sur la démographie des peuples depuis les mortalités dues aux combats jusqu'aux pathologies et aux épidémies de guerre les plus habituelles (famines, maladies de carence, choléra, grippe, tuberculose...). Lorsque la métaphore d'un front de guerre, qui serait partout et nulle part, est retenue pour poser la singularité de la guerre mondiale au $\mathrm{Xx}^{\mathrm{e}}$ siècle, il s'agit bien d'entrer dans une lecture de la guerre qui engage l'ensemble du corps de la société, jusque dans son esprit travaillé par les censures et les propagandes. Aussi la fin du héros militaire, dans les figures guerrières les mieux établies de l'histoire de la guerre en Occident, voisine avec le sacrifice du « soldat inconnu », métaphore du visage non figuré, voire défiguré, du combattant des guerres totales.

Aussi le débat historiographique, notamment sur l'interprétation de la guerre de 1914-1918, s'est-il attaché à comprendre pourquoi et comment les peuples avaient assumé la charge de la guerre, au sens où ils l'avaient massivement faite, y compris par les contraintes politiques et militaires qui n'excluaient pas des formes de contrôle social, avant de la refuser, mais sur sa fin ou une fois terminée. L'histoire des entrées et des sorties de guerre est une variante, une autre configuration de l'analyse des 
« unions sacrées » et des « Burgfrieden », qui a été préalablement et tout particulièrement appliquée aux deux conflits mondiaux car les peuples, entendons les opinions publiques, étaient devenus des acteurs majeurs de la guerre de masse?

La notion de nature de la guerre qui permettrait de différencier la guerre limitée (par les buts, moyens, armes et objectifs recherchés, selon Ian $\mathrm{Clark}^{8}$ ) de la guerre illimitée ou totale, ne fait pas consensus dans le débat des historiens du contemporain, pour ne pas éclaircir parfaitement le débat sur ses origines. La guerre illimitée est souvent confondue avec la guerre absolue sur le plan théorique. En dépit du fait que l'historien $\mathrm{du} \mathrm{Xx}^{\mathrm{e}}$ siècle revendique l'apparition de la notion de guerre totale pour le $\mathrm{xx}^{\mathrm{e}}$ siècle, les guerres de Trente Ans ou de Sept Ans, où Prusse et Autriche luttèrent avec toutes leurs forces y compris pour la survie pour la première, lui semblent pourtant répondre largement aux critères de la guerre totale. Le but d'une destruction totale de l'adversaire accomplirait le mieux la définition. Enfin, la notion de guerre limitée n'est pas apparue opératoire pour l'ère nucléaire, pas plus d'ailleurs que la définition n'est aisée pour les conflits conventionnels limités, antérieurs à l'ère de l'atome. Le débat s'est refermé par une définition de la guerre limitée comme stratégie d'usure de l'adversaire en opposition à la guerre totale comme stratégie d'anéantissement de l'ennemi, et ceci dès la guerre antique (Assyriens...).

La guerre totale, en supposant que l'on reprenne la notion pour les guerres de la fin du XVIII siècle, n'est-elle que le degré extrême d'une échelle, ou représente-t-elle un changement de nature et une autre essence de la guerre? Dans cette dernière hypothèse, l'année 1792 tout particulièrement - on peut élargir à 1792-1794 - représenterait une rupture. En quoi?

\section{David A. BeLL}

Je veux souligner ici la distinction entre une guerre totale et une guerre meurtrière, voire exterminatrice. Inutile de dire que l'humanité a eu son lot de conflits exterminateurs bien avant le $\mathrm{XVIII}^{\mathrm{e}}$ siècle et pendant celui-ci. La guerre totale est un phénomène beaucoup plus spécifique et plus original. À une échelle géographique limitée, elle est concevable à

(7) Jean-Jacques Becker, Gerd Krumeich, La Grande Guerre. Une histoire francoallemande, Paris, Tallandier, 2008.

(8) Ian Clark, Waging War. Philosophical Introduction, Oxford, 1988, p. 58-60. 
des époques reculées. Mais à l'échelle des grands états territoriaux, elle est quelque chose de strictement moderne.

Je pense que ma définition de la guerre totale comme phénomène politique est utile pour regrouper un certain nombre de conflits qui ont des choses importantes en commun, tout en rendant compte des origines du terme et de son usage dans la langue politique contemporaine. Dans mon livre La première guerre totale, j'ai essayé de repérer ses origines idéologiques dans l'Europe des Lumières, et de tracer le passage, en Europe occidentale, de la guerre aristocratique et réglée de l'Ancien Régime à la guerre totale qui commence en $1792^{\circ}$. Il est cependant possible d'accepter une définition politique de la guerre totale sans accepter cette explication de ses origines.

Peut-on alors considérer la guerre révolutionnaire et napoléonienne comme une guerre totale? Oui, si l'on prend au sérieux une définition politique de la guerre totale. Malgré les capacités matérielles qui n'ont pas beaucoup évolué depuis plusieurs décennies, les guerres de la Révolution et de Napoléon Bonaparte ont des buts et connaissent une radicalisation sans précédent dans l'histoire européenne. À la différence des guerres de l'Ancien Régime, après 1792, les belligérants des deux côtés visent non seulement la défaite de l'ennemi mais aussi le renversement de son gouvernement. Ils imaginent aussi une mobilisation totale de la société. Il va sans dire qu'ils n'emploient pas le terme de guerre totale mais, dans la proclamation de la levée en masse de 1793, ainsi que dans le Corso terrestre espagnol de 1809 ou dans la déclaration sur la Landsturm prussienne de 1813, l'idée d'une mobilisation de toutes les ressources humaines et matérielles d'une société pour la guerre est esquissée de façon très claire ${ }^{10}$. Dans la propagande diffusée par la France et dans celle de ses ennemis, on voit aussi la diabolisation de l'ennemi, voire sa transformation en « ennemi du genre humain »-hostis humani generis $^{11}$ - . Mis ensemble, ces différents éléments idéologiques signifient pour les guerres révolutionnaires et napoléoniennes ce que le terme de guerre totale signifie pour la fin de la première guerre mondiale et pour la deuxième guerre mondiale.

(9) Voir David A. BeLL, La première guerre totale... op. cit., p. 62-96.

(10) Ibid., p. 299-342.

(11) Ibid., p. 137-174 et p. 299-342; Dan Edelstein, The Terror of Natural Right: Republicanism, the Cult of Nature and the French Revolution, Chicago, University of Chicago Press, 2009. 


\section{Hervé DréviLLON}

Toute la question se situe là. L'usage du concept de guerre totale mélange deux acceptions: degré extrême dans un échelonnement et essence particulière de la guerre. Ces deux définitions sont hétérogènes et ne convergent que sur certains points. Il en résulte une confusion extrême qui alimente l'impression que la guerre totale se dérobe toujours quand on veut la saisir avec rigueur et précision. Quant à la question particulière de la guerre totale comme essence particulière de la guerre, elle soulève elle-même un problème de cohérence. Si on se situe dans la perspective clausewitzienne, il faut alors parler de " guerre absolue », c'est-à-dire la réalisation idéale du concept de guerre, ce qui suppose une double rupture politique : participation de la nation à la guerre (1792-1794) et organisation d'une forme politique de direction de la guerre, que Clausewitz limite à l'Empire. Dans ce cas, c'est l'ensemble Révolution Empire qui fait la rupture. Mais si on se place dans la perspective de Ludendorff, l'essence de la guerre totale réside dans la soumission du politique au militaire, ce qui ne survient jamais pendant la Révolution, ni sous l'Empire. Une grande ambiguïté résulte du fait que le concept de guerre totale appartient à Ludendorff, alors que le scénario décrit par les historiens qui l'utilisent est clausewitzien. Or, on ne peut pas être «ludendorffien » et « clausewitzien » à la fois...

\section{Olivier ForCADE}

Nouvelle guerre ou autre guerre? Dans la sociologie militaire américaine depuis 1945 et dans les travaux anglo-saxons d'histoire militaire, les auteurs ont parlé, tour à tour, de guerre « totale », « grande » ou « très grande », " industrielle » ou plus récemment «majeure », avant que le brouillage des catégories d'analyse des formes de conflits infra-nucléaires après la guerre froide conduise à substituer des notions inédites qui prennent en compte la difficulté de repérer, en historien ou en politiste, le passage de la paix à la guerre ${ }^{12}$. La notion de "guerre absolue » est ici moins prégnante dans le débat contemporain, différemment mobilisée dans le vocabulaire de guerre qui a désigné une expérience de l'indicible davantage que de l'absolu.

La guerre totale, si elle est définie comme une guerre d'anéantissement des forces militaires et d'une société dans ses fondements et son

(12) Frédéric Ramel, Jean-Vincent Holeindre (dir.), La fin des guerres majeures, Paris, Economica, 2010. 
existence, est un basculement dans une autre forme de guerre. Il ne s'agit pas seulement d'un changement de seuil, mais bientôt d'essence. Par exemple, le bombardement était déjà l'objet d'une tentative de réglementation lors de la conférence de La Haye de 1907. Bien sûr, le bombardement par l'artillerie lourde dans la guerre de 1914-1918 et son utilisation orientée contre les populations civiles sur le front occidental, à compter de l'automne 1916, retouche un paramètre tactico-opérationnel de la guerre. Entre fin mars et début mai 1918, vingt-trois bombardements eurent lieu sur Paris, faisant fuir 800000 des 3 millions de Parisiens en six mois. Or, le bilan montrait des pertes humaines très faibles rapportées à celles de la guerre, soit 267 tués et 602 blessés, mais avec des conséquences psychologiques fortes. L'expérience du bombardement aérien par des pièces à longue portée installées dans l'Aisne, à une distance de $140 \mathrm{~km}$ de la cible, ouvrait une nouvelle page de la guerre. Il faut donc progressivement parler d'une conception stratégique nouvelle de l'emploi (massif) de l'artillerie lourde, utilisée ponctuellement contre des populations civiles et les villes ${ }^{13}$.

Puis, de façon systématisée sinon théorisée dans la guerre aérienne contre les villes dans les années 1930 aux États-Unis notamment, le bombardement stratégique, en 1939-1945 confirme, pour l'essentiel, un changement de seuil davantage que d'essence de la guerre ${ }^{14}$. Le bombardement stratégique est au cœur de la stratégie aérienne. Tout à la fois « symbole et manifestation de la puissance aérienne, il est la marque de la guerre totale $»^{15}$. Décisive, l'entrée dans l'âge stratégique nucléaire est moins un changement de seuil que de nature et d'essence de la guerre. Le bombardement nucléaire pose une autre question à la stratégie, sur la potentialité technologique et la possibilité pratique d'une destruction totale d'un pays et de la vie humaine. Au début de la guerre froide, les débats sur la stratégie nucléaire valorisent l'idée de la dissuasion au détriment de l'action en même temps qu'ils invitent à dépasser la guerre.

(13) Philippe Chassaigne, Jean-Marc Largeaud (dir.), Villes en guerre (1914-1945), Colin, 2004 ; J. Konvitz, « Représentations urbaines et bombardements stratégiques », Annales ESC, juilletaoût 1989, 44 année, ${ }^{\circ}$ 4, p. 823-848; Patrick FACON, Le bombardement stratégique, Monaco, Éd. du Rocher, 1996; Danièle Voldmann, « Les populations civiles, enjeux du bombardement des villes (1914-1945), Stéphane Audoin-Rouzeau et alii (dir.), La violence de guerre 1914-1945. Approche comparée des deux conflits mondiaux, Complexe, 2002.

(14) Hervé Coutau-BÉGarie, Traité de stratégie, Economica, 2006 ( $5^{\mathrm{e}}$ éd.), p. $743 \mathrm{sq}$; Serge Gadal, La guerre aérienne vue par William Sherman, Economica, 2006.

(15) Hervé Coutau-BÉGarie, Traité de stratégie...op. cit., p. 743. 
Si on considère au contraire qu'elle est une étape dans la gradation d'un processus, y a-t-il un critère unique pour situer le curseur? Ne fautil pas plusieurs critères et lesquels? Par exemple, le processus de formation d'un complexe militaro-industriel, qui est fondamental pour les théoriciens de la guerre totale $d u X X^{e}$ siècle, est déjà bien présent lorsque commencent les guerres révolutionnaires; avec les arsenaux, avec la standardisation de l'armement, toutes évolutions présentes dès le règne de Louis XIV.Alors à quel moment se situerait la rupture dans les domaines de l'armement, de la logistique, etc...?

\section{David A. BELL}

Dans le dernier volume de la série des colloques et volumes collectifs cités plus haut ${ }^{16}$, qui porte précisément sur l'époque révolutionnaire, une partie des auteurs avance l'idée que les guerres de 1792 à 1815 sont une guerre totale mais une autre partie rejette cette conception et le terme ne paraît pas dans le titre du livre.

Cette confusion et ce désaccord sont dus principalement au fait que ces historiens considèrent la guerre surtout du point de vue des capacités matérielles des sociétés belligérantes. C'est-à-dire qu'ils veulent distinguer les guerres totales des autres guerres par la technologie, par les structures économiques et industrielles et par l'organisation militaire. Plusieurs d'entre eux soutiennent que la guerre totale n'est rendue possible que par la révolution industrielle - par le développement des moyens de mobiliser et d'armer la quasi-totalité d'une population masculine adulte et de la transporter rapidement vers les champs de bataille. Et aussi par le développement des moyens de livrer une campagne d'extermination à une échelle industrielle contre une population civile ennemie. C'est d'ailleurs le point de vue de la plupart des historiens qui ne croient pas que les guerres révolutionnaires puissent être considérées comme des guerres totales ${ }^{17}$. Du point de vue de l'organisation matérielle, elles sont bien sûr plus proches de celles de Louis XIV que des guerres mondiales. Les armes utilisées alors, ainsi que les moyens de les fabriquer, n'auraient pas été étrangères aux combattants de la Guerre de la Succession d'Espagne, il en est de même pour la tactique, voire pour les ratio de mobilisation. Selon ces critères matériels, les guerres européennes de 1792 à 1815 n'appartiennent pas à l'époque des guerres totales mais à celle des mousquets, du canon

(16) Voir note 1.

(17) Voir aussi et surtout Michael BroERs, « The Concept of 'Total War' in the RevolutionaryNapoleonic Period » War in History, vol. 15, no. 3, 2008, p. 247-68. 
et de la marine à voile. La césure entre les deux époques de la guerre se trouverait donc ailleurs, en aval de Waterloo.

Cependant, les distinctions au niveau des capacités matérielles ne sont pas aussi claires qu'il semble à première vue. D'où la nécessité d'une définition politique de la guerre totale. Toutefois, celle-ci ne néglige pas les facteurs matériels. Une tentative sérieuse de mobiliser toute une société - chaque être humain, chaque ressource - à une large échelle géographique ne peut s'imaginer sans l'existence d'un état doué de grandes capacités administratives. Il faut pouvoir établir un système efficace de conscription, diriger les manufactures d'armes et de munitions, assurer la fourniture des armées, etc. Ainsi, la Guerre de Trente Ans, malgré de terribles pertes humaines, n'est pas une guerre totale car elle ne connaît pas de tentative de mobilisation totale. Elle ne connaît pas non plus une dynamique de radicalisation irrésistible.

\section{Hervé Drévillon}

Il n'existe aucun critère quantitatif permettant de repérer une quelconque rupture. Les critères généralement admis : pertes lors des batailles, nombre total des morts, ratio nombre de soldats/population globale, mobilisation économique, etc. ne sont ni convergents, ni concluants. C'est la raison pour laquelle, l'application du concept de guerre totale aux guerres de la Révolution ou de l'Empire résulte, le plus souvent, d'une pesée globale dont les composantes sont mal distinguées et mal singularisées.

\section{Olivier Forcade}

La question de la mobilisation économique dans la guerre totale introduit à l'idée d'un processus de mobilisation industrielle, technologique et financière totalement accompli qui opère une conversion radicale d'une économie civile en économie de guerre : innover, armer, produire dans la seule logique de la guerre. Le retour de l'économie de guerre à l'économie civile met en évidence ce processus historique. Au-delà de l'économie, la société est affectée par cette transformation jusque dans son organisation et dans le rôle des sexes. C'est la mobilisation de la nation, prévue y compris dès le temps de paix, qui fait prendre en compte la préparation d'un pays à la guerre totale. La loi de 1938 sur la mobilisation de la nation en France est exemplaire ici, comme le rappelle un colloque récent ${ }^{18}$. La notion de « système d'arme » signalé par les histo-

(18) Dominique BARJOT (dir.), La mobilisation de la nation à l'époque de la guerre totale, Economica, à paraître. 
riens de la guerre au XVII à la suite d'André Corvisier, Jean Bérenger revenant sur le débat historiographique consacré à la révolution militaire aux $\mathrm{XV}$-XVIII ${ }^{\mathrm{e}}$ siècles, Jean Chagniot et des spécialistes d'histoire de l'armement, des sciences et des techniques à l'instar de Dominique Pestre et de Patrice Bret ${ }^{19}$, est utile pour repérer la généalogie de la guerre totale.

Ce processus d'échelonnement, de l'Ancien Régime à la période révolutionnaire et impériale, puis au cours des deux derniers siècles, est-il irréversible? Y a-t-il des seuils?

\section{David A. BELL}

Dans les guerres de 1792 à 1815 , une image de la mobilisation totale d'une société en vue de la défaite d'un ennemi diabolisé se constitue très tôt et mène à une radicalisation incontrôlable de la guerre. Il est vrai que Napoléon Bonaparte, une fois arrivé au pouvoir, tente souvent de freiner cette radicalisation, et abandonne largement la rhétorique jacobine d'un conflit apocalyptique entre la France et ses ennemis. S'il avoue à Benjamin Constant en 1815 que « je voulais le sceptre du monde; et qui ne l'eût pas désiré à ma place? », il proclame encore plus souvent qu'il veut faire la paix et prendre sa place parmi ses souverains frères ${ }^{20}$. Cependant, et voici le point essentiel, Bonaparte ne réussit jamais dans ce projet de mener une guerre limitée. Au contraire, c'est maintenant le tour de ses adversaires de diaboliser l'envahisseur français et de proclamer la guerre totale contre lui. Les tentatives successives de paix échouent et l'échelle des batailles ne cesse de croître. Avant 1790, une poignée de batailles seulement engage plus de 100000 combattants; en 1809, la bataille de Wagram, la plus grande jamais vue depuis l'apparition de l'arme à feu, en implique 300000 . Quatre ans plus tard, la bataille de Leipzig en compte 500000 dont 150000 tués ou blessés ${ }^{21}$. Les guerres ne se terminent pas par des négociations entre des puissances qui se reconnaissent mutuel-

(19) André CoRvisier (dir.), Dictionnaire d'art et d'histoire militaires, PUF, 1988; Ibid., Histoire militaire de la France, PUF, 1992-1994; Jean СнаGNiot, Guerre et société à l'époque moderne, PUF, 2001; Jean BÉRENGER (dir.), La Révolution militaire en Europe XV-XVIII siècles, Economica, 1998; Patrice Bret et Dominique Pestre (dir.), Deux siècles d'histoire de l'armement en France. De Gribeauval à la force de frappe, CNRS, 2005.

(20) Napoléon Bonaparte, cité dans William Henry IRELand, Anecdotes sur Napoléon, Et ses entretiens, Paris, chez l'éditeur, 6-8 bd. du Temple, 1823, p. 91. Voir à ce sujet Annie Jourdan, « Napoléon et la paix universelle : Utopie et réalité », Napoléon et l'Europe, Colloque de la Rochesur-Yon, Jean-Clément Martin (dir.), Rennes, Presses Universitaires de Rennes, 2002, p. 55-69.

(21) Voir David A. Bell, La première guerre totale, op. cit., p. 12. 
lement comme légitimes et honorables mais par l'effondrement de la France en 1814, et l'emprisonnement de son empereur comme s'il était un criminel.

À plusieurs moments des guerres de 1792 à 1815 , l'intensité des combats et le manque de contrainte qui sont souvent associés avec le concept de guerre totale semblent absents. Cependant, il en est de même pour toutes les guerres, la deuxième guerre mondiale y comprise. Pensons à la « drôle de guerre » de 1939-40, par exemple. Ou au traitement des prisonniers allemands aux États-Unis, qui ressemble beaucoup plus aux pratiques européennes du dix-neuvième siècle qu'à celles des Nazis envers les prisonniers soviétiques (dans le Mississippi, les commandants allemands ont même le droit d'aller au cinéma ${ }^{22}$ ). Encore une fois, ce qui compte dans la guerre totale c'est la radicalisation, la dynamique politique qui pousse les belligérants vers un engagement total et vers l'abandon de toute contrainte. Et de ce point de vue, les guerres de 1792 à 1815 , aussi différentes qu'elles soient des guerres mondiales $\mathrm{du} \mathrm{xx}^{\mathrm{e}}$ siècle, constituent néanmoins la première guerre totale.

$\mathrm{Si}$, selon cette définition politique, les deux guerres mondiales sont des guerres totales, ainsi qu'au cours du $\mathrm{XIX}^{\mathrm{e}}$ siècle la guerre civile américaine de 1861 à 1865 , la guerre franco-prussienne ne l'est pas car les belligérants ont des buts nettement plus limités et le processus de radicalisation incontrôlable n'est pas engagé.

Existe-t-il un seul « seuil » technologique ou industriel qui peut distinguer les « guerres totales » des autres guerres? On pourrait soutenir que les différents aspects de la guerre possèdent des seuils différents. Par exemple, pour tuer ou blesser plus de cent cinquante mille soldats au cours d'une seule bataille, les armes à feu sont parfaitement suffisantes, comme en témoigne la bataille de Leipzig de 1813. Pour massacrer une population civile ennemie, une technologie encore plus primitive suffit largement (Carthago delenda est). Mais pour atteindre rapidement et de loin une grande partie de la population ennemie, sans qu'elle ait la possibilité de fuir, il ne faut pas simplement la guerre industrielle mais la guerre aérienne. Et selon ce critère, même la première guerre mondiale (qui d'ailleurs épargne largement les populations civiles des grandes puissances) n'est pas une guerre totale. La guerre exterminatrice par excellence, c'est-à-dire la guerre atomique, ne serait pas non plus une

(22) Voir John RaY SKates, "German Prisoners of War in Mississippi, 1943-1946," Mississippi History Now, septembre 2001, http://mshistory.k12.ms.us/index.php?id=231. 
guerre totale, car elle n'exigerait pas une mobilisation à grande échelle des ressources économiques et humaines de la part des combattants. Les États-Unis se servent de la bombe atomique en 1945 précisément pour éviter la mobilisation extrême qui aurait été nécessaire pour envahir le Japon.

Il est donc extrêmement difficile de définir « la guerre totale » en termes matériels.

\section{Hervé Drévillon}

La question des seuils a fait l'objet de nombreux débats historiographiques, qui restent toujours ouverts. La diversité des usages du concept de guerre et de son application oblige l'historiographie à inventer autant de critères qu'il y a d'exemples de guerres dites totales.

\section{Olivier ForCADE}

Les historiens de la guerre 1914-1918 ont fait valoir que ce conflit faisait entrer dans une autre guerre, dans la guerre majeure par les franchissements sans précédent des seuils de violence, non seulement sur le champ de bataille mais également contre les populations civiles; d'autre part, les armées alignent massivement des populations hier civiles, aujourd'hui sous uniforme et combattant aux côtés et encadrées par les armées professionnelles. L'idée d'une montée aux extrêmes renvoie bien à l'observation d'une société luttant pour la survie de son existence, sinon de la civilisation, face à un ennemi qui menace l'intégrité même de son existence. Ainsi le débat entre les historiens du $\mathrm{Xx}^{\mathrm{e}}$ siècle est-il allé jusqu'à caractériser un " âge des extrêmes ». La notion n'a pourtant pas toujours été comparée à toutes les époques et toutes les civilisations.

D'autre part, le débat a tendu à se polariser depuis vingt ans sur l'étude des conflictualités et de la violence de guerre, dans des échanges entre les sciences humaines et sociales, précisément parce que la frontière entre le temps de paix et de guerre pour définir les formes de la conflictualité post-guerre froide constituait pour l'historien une difficulté de méthode, sinon d'objet. De fait, les notions de violence de guerre et de seuil de violence ont élargi le débat à une anthropologie de la guerre qui se saisissait de l'histoire de la guerre par les violences faites au corps. Dès lors, l'analyse des formes du combat et de la destruction du corps, portant la négation de l'esprit humain, a orienté les travaux des historiens de deux guerres mondiales. Cette disparition de la preuve et de la trace de la guerre sur un corps dilacéré, volatisé dans le combat d'artillerie, ouvrait à une autre idée de la destruction de l'indi- 
vidu et, partant, de l'humanité. L'homme n'est pas seulement mort, il a, au propre et au figuré, physiquement disparu sur le champ de bataille, sinon du champ de bataille. Peut-être y a-t-il là une autre approche de la guerre totale, qui rend inutile au combat même la présence de l'homme; ce dernier a cessé d'être l'acteur central de la guerre pour n'en être plus que la victime.

L'idée d'un processus irréversible est difficile à accepter sinon à concevoir car le passage de seuils vers l'accomplissement de la guerre totale permet de concevoir la montée aux extrêmes comme l'éloignement des extrêmes, en «redescendant» les seuils franchis de la destruction et de l'anéantissement. La guerre nucléaire n'a pas eu lieu après 1945 et l'après-guerre froide a laissé la place à des conflits dont les formes éloignaient la guerre totale pour y substituer la guerre " asymétrique », « révolutionnaire », " irrégulière $»^{23} \ldots$ Dans la communauté internationale (l'expression est-elle préférable à celle de «l'Occident»?), le débat actuel sur l'insurrection et la contre-insurrection répond à d'autres définitions qui satisfont à des buts différents de la guerre de pacification, au maintien ou à l'imposition de la paix.

Guibert, dans le Système de la guerre moderne, explique que si on fait participer l'ensemble de la Nation à la guerre, alors celle-ci deviendra illimitée. Ce qui pose la question de la guerre de masse. Mais, si on regarde les ratio de mobilisation à la fin du XVII siècle et ceux de la fin du XVIII siècle, ils sont pratiquement identiques, pour nous en tenir à des contextes comparables. L'impression d'un changement d'échelle n'estelle pas une illusion?

\section{David A. Bell}

Certes, ces ratio sont identiques, on l'a vu plus haut, de même que bien des traits de l'organisation matérielle. Mais au regard d'une définition politique de la guerre totale, les similitudes et les différences entre ces types de guerre sont tout autres.

\section{Hervé DréviLLoN}

Oui, absolument. La question des seuils quantitatifs n'étant pas décisive, il faut convenir que l'on se situe dans l'illusion d'un changement d'échelle. On peut dès lors se demander ce qui entretient cette illu-

(23) Gérard Chaliand, Les guerres irrégulières XX-XXI siècle, Folio-Histoire, 2008, p. 17-64 notamment. 
sion si ce n'est pas le nombre. En réalité, cette illusion résulte de la nature politique de la guerre. Guibert, dans le traité de la force publique situe la question non pas au niveau quantitatif de la mobilisation de toute la population, mais au niveau politique. Il s'oppose à la mobilisation de la garde nationale comme force armée extérieure, ce qui signifierait, selon lui, l'engagement du corps politique dans la guerre et créerait ainsi une confusion entre le civil et le militaire. Ainsi, il ne suffit pas que la nation soit mobilisée dans l'effort de guerre pour que celle-ci devienne totale; il faut que cette mobilisation emprunte les voies d'un enrôlement militaire du corps politique.

Toujours dans l'hypothèse d'une transformation des formes et de la nature des conflits à la fin du XVIII siècle, ne faut-il pas comprendre celleci dans un vaste espace atlantique (englobant l'Amérique du Nord pendant la Guerre de Sept Ans, puis la Guerre d'Indépendance américaine, l'agitation en Irlande et aux Provinces-Unies), et ne pas se limiter au seul contexte révolutionnaire français, aussi important soit-il?

\section{Hervé DréviLlon}

On trouve bien sûr des formes d'accroissement de l'intensité du conflit lors de la guerre de Sept Ans : traitement des prisonniers (sur mer), déportation des Acadiens, accroissement des taux de pertes dans les batailles (terrestres et navales), etc. Mais en se plaçant sur ce terrain de l'évaluation de l'intensité des guerres, on ne fait que déplacer le problème de la guerre totale et de l'incohérence de ce concept.

La dichotomie classique à travers laquelle on présente le passage d'une guerre réglée, soi-disant aristocratique, à une guerre totale, n'estelle pas une vue de l'esprit? Ou du moins un point de vue très limité à l'Europe occidentale, qui n'a aucun sens pour les espaces coloniaux, pour l'Europe orientale, et même pour les marges des grands États européens (Écosse, Irlande, espaces méditerranéens)?

\section{David A. BELL}

De même que la Guerre de Trente Ans n'était pas une guerre totale, il en va de même pour les guerres menées au dix-huitième siècle dans les espaces coloniaux et en Europe orientale, même si le comportement des combattants dans ces conflits, surtout envers des civils, aurait été fortement condamné par les théoriciens de la guerre réglée des états aristocratiques occidentaux. 


\section{Hervé DréviLlon}

Les ambiguïtés du concept de guerre totale existent également pour celui de guerre réglée (ou guerre limitée). La guerre réglée est-elle une essence ou un degré de la guerre? Faute de pouvoir répondre à cette question, on ne peut déterminer quelles guerres étaient réglées ou pas.

David BeLL Université de Princeton dabell@princeton.edu

Annie CREPIN crepinannie@neuf.fr

Hervé DREviLLon Université de Paris I Pôle Histoire et Défense IRSEM hv.drevillon@wanadoo.fr

Olivier ForCADE Université de Paris IV forcade.olivier@neuf.fr

Bernard GAINOT Université de Paris I bgainot@orange.fr 